\title{
Health-Related Quality of Life of Patients with Intermediate Hepatocellular Carcinoma after Liver Resection or Transcatheter Arterial Chemoembolization
}

\author{
ZR Xie, YL Luo*, FM Xiao, Q Liu, Y Ma
}

\begin{abstract}
Background: The aim of our present study was to compare quality of life $(\mathrm{QoL})$ between intermediate-stage (BCLC-B) HCC patients who had undergone either liver resection or transcatheter arterial chemoembolization (TACE). Materials and Methods: A total of 102 intermediate-stage HCC patients participated in our study, including 58 who had undergone liver resection and 44 who had undergone TACE. Baseline demographic characteristics, tumor characteristics, and long-term outcomes, such as tumor recurrence, were compared and analyzed. QoL was assessed using the Short Form (SF)-36 health survey questionnaire with the mental and physical component scales (SF-36 MCS and PCS). This questionnaire was filled out at HCC diagnosis and 1, 3, 6, 12, 24 months after surgery. Results: For the preoperative QoL evaluation, the 8 domains related to QoL were comparable between the two groups. The PCS and MCS scores were significantly decreased in both the TACE and resection groups at1 month after surgery, and this decrease was greater in the resection group. These scores were significantly lower in the resection group compared with the TACE group $(P<0.05)$. However, these differences disappeared at 3 and 6 months following surgery. One year after surgery, the resection group showed much higher PCS scores than the TACE patients $(P=0.018)$, and at 2 years after surgery, the PCS and MCS scores for the resection group were significantly higher than those for the TACE group $(P<0.05)$. Eleven patients $(\mathbf{1 9 . 0 \%})$ in the resection group and $17(38.6 \%)$ in the TACE group suffered HCC recurrence $(P<0.05)$. Univariate and multivariate analyses indicated that tumor recurrence $(H R=1.211,95 \% \mathrm{CI}: 1.086-1.415, P=0.012)$ was a significant risk factor for poorpostoperative QoL in the HCC patients.Conclusions: Due to its effects on reducing $\mathrm{HCC}$ recurrence and improving long-term QoL, liver resection should be the first choice for the treatment of patients with intermediate-stage HCC.
\end{abstract}

Keywords: Hepatocellular carcinoma - quality of life - liver resection - transcatheter arterial chemoembolization

Asian Pac J Cancer Prev, 16 (10), 4451-4456

\section{Introduction}

Hepatocellular carcinoma (HCC) is the fifth most common cancer and the third most frequent cause of mortality in cancer patients (Jemal et al., 2011). Moreover, the burden of HCC is greater in China due to the high prevalence of hepatitis B virus (HBV) infection in this country (Maluccio et al., 2012). Radiofrequency ablation (RFA), liver resection, and liver transplantation represent the mainstay of radical therapy for early stage HCCs, and transcatheter arterial chemoembolization (TACE) is considered an alternative treatment for unresectable HCC (Bruix et al., 2014; Zuo et al., 2015). According to the Barcelona Clinic Liver Cancer (BCLC) HCC staging system, TACE should be considered for patients with BCLC-B (intermediate-stage) HCC, and hepatic resection is only recommended for patients with early-stage HCC and satisfactory liver function (BCLC-A) (Bruix et al., 2011; Xu et al., 2014). BCLC-B stage includes Child A and $\mathrm{B}$ patients with multifocal $\mathrm{HCC}$, which is defined as $>3$ tumors of any size or 2-3 tumors with a maximal diameter of $>3 \mathrm{~cm}$ and the absence of vascular invasion or extrahepatic spread (Marrero et al., 2005). However, many studies have indicated that patients with intermediate-stage HCC may also benefit from radical hepatic resection with comparable long-term outcomes (Jianyong et al., 2014; Zhong et al., 2014). Therefore, controversy still exists regarding the choice of treatment for intermediate-stage HCC. However, although there have been many reports on long-term survival andtumor recurrence for patients receiving these therapies, no comparisons of their quality of life (QoL) have been performed.

In recent years, QoL has been proven to be a valuable parameter and is considered to be as important as overall survival and tumor-free survival for cancer patients (Slevin et al., 1992; Han et al., 2014). In fact, the multimodal concept of QoL has been used in many medical practices (Langenhoff et al., 2001; Scurtu et al., 2005). Moreover, QoL has become an important consideration when selecting an optimal treatment. Some studies have 
evaluated the effects of treatment modalities on healthrelated QoL in HCC patients (Yeo et al., 2006; Fan et al., 2010; Diouf et al., 2013; Gandhi et al., 2014; Diouf et al., 2015). In addition, some researchers have discussed the impact of liver resection on postoperative QoL (Bruns et al., 2010; Mise et al., 2014), and others have studied QoL after TACE (Wang et al., 2007; Xing et al., 2015). Moreover, another report has compared the QoL outcomes for RFA, liver resection and TACE (Toro et al., 2012) and has found that hepatic resection provides the best QoL at 24 months compared with TACE or RFA. However, in this previous study, HCC was not graded according to tumor characteristics, such as tumor number, size, and liver function.

Therefore, in our present study, a retrospective QoL assessment was conducted to evaluate intermediate-stage HCC patients who had undergone liver resection or TACE as aninitial treatment. The aim of this study was to assess the impacts of surgical protocols on postoperative QoL in patients with BCLC stage B HCC.

\section{Materials and Methods}

Patients who were diagnosed with HCC from Jan 2009 to Feb 2013 at the department of liver surgery, West China Hospital of Sichuan University were included in our present study. All of these patients had undergone liver resection or TACE as aninitial therapy for HCC. The inclusion criteria were as follows: between 18 and 60 years of a geat the time of HCC diagnosis; BCLC stage B HCC; Child A or B liver function;multifocal HCC, defined as $>3$ tumors of any size or 2-3 tumors with a maximal diameter of $>3 \mathrm{~cm}$; the absence of vascular invasion or extrahepatic spread; having undergone liver resection or TACE as aninitial therapy; and no contraindication for surgery. The exclusion criteria were as follows: Child $\mathrm{C}$ liver function; BCLC stage A or C HCC; having undergone other procedures, such as liver transplantation or RFA; loss to follow-up; and inability to complete the questionnaire due to illiteracy. The present study conformed to the ethical guidelines of the Declaration of Helsinki and was approved by the ethics committee of our hospital. All of the patients provided informed consent.

Based on the inclusion and exclusion criteria, $102 \mathrm{HCC}$ patients were included in this study. Moreover, we divided the patients into two groups based on the initial therapy, the liver resection group (58 patients) and the TACE group (44 patients). All of these patients completed the Medical Outcomes Study Short Form-36 (SF-36) questionnaire pre-operatively and at 1, 3, 6, 12, and 24 months after surgery. We then compared the baseline demographics of these patients, such as age, gender, body mass index (BMI), underlying disease, liver function (Child score), liver cirrhosis, and tumor characteristics (i.e., tumor diameter, tumor number, alpha-fetoprotein (AFP) level, and tumor differentiation). We also compared the mental and physical component scores of the questionnaires completed at the above time points.

Health-related QoL was assessed using the validated Chinese version (2002) of the SF-36 (Li et al., 2002; Jin et al., 2010). The SF-36 is a validated, self-administered questionnaire that is used internationally to measure the following 8 domains of health: general health, bodily pain, social functioning, role-physical, physical functioning, vitality, role-emotional, and mental health. The raw scores of each questionnaire were converted to scores ranging from 0 to 100, with higher scores indicating higher levels of functioning or well-being (Jin et al., 2010). QoL was compared between the two groups at the different time points. Scores representing overall physical and mental functioning were presented as physical component summary scale (PCS) and mental component summary scale (MCS) scores (Jin et al., 2010). The PCS, which is primarily based on the physical functioning, rolephysical, bodily pain, and general health components, reflects apatient's overall physical health status. The MCS is basedprimarilyon the vitality, social functioning, roleemotional, and mental health components and reflects a patient's overall mental health status.

All of the hepatic resection and TACE procedures have been described in our previous studies (Jiang et al., 2014; Zhang et al., 2014). All of these procedures were conducted by surgeons with over 20 years of hepatic resection and TACE experience. The efficacy of surgery was evaluated at 1 month by contrast-enhanced CT or MRI and according to the levels of tumor markers (AFP). Thereafter, efficacy was measured every 2 to 3 months over the following yearand every 5-6 months from that point forward to check for recurrence. Chest radiography and bone scintigraphy were performed when extrahepatic HCC recurrence wassuspected. Liver function was another key post-operative factor that was assessed.

\section{Statistical analysis}

Data were obtained by surgeons and nurses and analyzed by a biostatistics expert. All of the data were collected and analyzed using the SPSS statistical software package (SPSS Inc., Chicago, USA; version 17.0). Descriptive statistics were calculated to provide information regarding demographic and disease-specific characteristics. Comparisons between the two groups with respect to QoL were performed using independent-sample Student's t-test, analysis of variance, or a non-parametric test, if necessary. A $P$ value of $<0.05$ was considered significant andthat of $<0.001$ was considered highly significant.

\section{Results}

\section{Patients' baseline and tumor characteristics}

As shown in Table 1, the baseline demographic characteristics were compared between the two groups. No significant differences were observed between the resection and TACE groups in terms of age, gender, or BMI. HCC was caused by HBV infection in most patients $(89.7 \%$ in the resection group and $90.9 \%$ in the TACE group, $P=0.582$ ). We also compared the patients' marital statuses, educational backgrounds, occupations and incomes because these factors may affect QoL after surgery and found no significant differences in these factors between the two groups. Although the liver resection group was found to have a lower monthly 
Table 1. Baseline Characteristics of the Patients, Tumors and Recovery for the Two Groups

\begin{tabular}{|c|c|c|c|}
\hline Patients' Baseline Characteristics & $\begin{array}{l}\text { Resection group } \\
\mathrm{n}=58\end{array}$ & $\begin{array}{c}\text { TACE group } \\
\mathrm{n}=44\end{array}$ & $P$ value \\
\hline Age & $46.9 \pm 8.3$ & $44.0 \pm 10.6$ & 0.123 \\
\hline Gender (M:F) & $48 / 10$ & $38 / 6$ & 0.622 \\
\hline BMI (kg/m2) & $23.0 \pm 2.2$ & $23.1 \pm 2.2$ & 0.766 \\
\hline Liver cirrhosis (HBV/HCV/no) & $52 / 2 / 4$ & $40 / 2 / 2$ & 0.582 \\
\hline Marital status (yes/no/divorced) & $53 / 2 / 3$ & $41 / 2 / 1$ & 0.975 \\
\hline Education (elementary/middle/university) & $15 / 29 / 14$ & $10 / 21 / 13$ & 0.55 \\
\hline Occupation (Farmer/worker/servant/other) & $14 / 21 / 13 / 10$ & $9 / 21 / 9 / 5$ & 0.67 \\
\hline Income (low/median/high) & $21 / 28 / 9$ & $7 / 29 / 8$ & 0.069 \\
\hline \multicolumn{4}{|l|}{ Tumor characteristics } \\
\hline Tumor target number & $2.3 \pm 1.4$ & $2.1 \pm 1.2$ & 0.506 \\
\hline Tumor diameter per patient & $7.6 \pm 1.8$ & $7.7 \pm 2.3$ & 0.665 \\
\hline AFP level & $730.4 \pm 512.9$ & $1688.3 \pm 5216.5$ & 0.167 \\
\hline \multicolumn{4}{|l|}{ Postoperative recovery } \\
\hline Complications (0/I/II/III/IV) & $36 / 11 / 9 / 2 / 0$ & $27 / 6 / 11 / 0 / 0$ & 0.843 \\
\hline Hospital stay days & $8.2 \pm 2.3$ & $5.1 \pm 1.0$ & $<0.001$ \\
\hline New targets (recurrence or metastasis, yes/no) & $11 / 47$ & $17 / 27$ & 0.028 \\
\hline
\end{tabular}

*Income (dollars/month), low: $\leq 500$; median: 500-800; high: $\geq 800$

Table 2. Tumor Recurrence According Tofollow-Up Time

\begin{tabular}{|c|c|c|c|c|c|}
\hline Groups & 0-1 months & 1-3 months & 3-6 months & 6-12 months & $12-24$ months \\
\hline Liver resection $(\mathrm{n}=58)$ & 0 & $1(1.7 \%)$ & $1(1.7 \%)$ & $4(6.9 \%)$ & $5(8.6 \%)$ \\
\hline TACE $(n=44)$ & 0 & $1(2.3 \%)$ & $3(6.8 \%)$ & $6(13.6 \%)$ & $7(15.9 \%)$ \\
\hline Overall & 0 & $2(2.0 \%)$ & $4(3.9 \%)$ & $10(9.8 \%)$ & $12(11.8 \%)$ \\
\hline
\end{tabular}

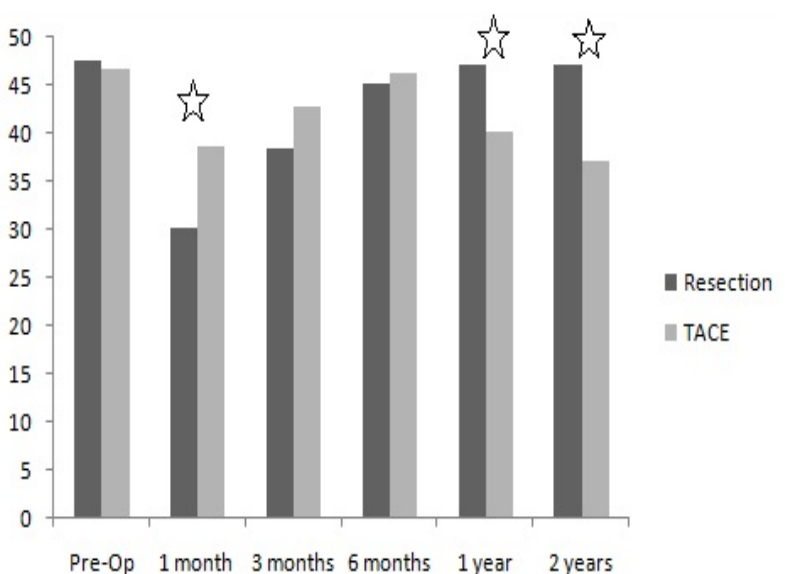

Figure 1. The PCS Scores were Comparable between the two Groups at Baseline. These scores were significantly decreased in both the TACE and resection groups at 1 month after surgeryand were significantly lower in the resection group compared withthe TACE group $(P<0.05)$. However, opposite results were observed between the two groups at 1 and 2 years after surgery (indicated by the stars, $P<0.05$ )

income, this difference was not significant $(P=0.069)$.

Tumor characteristics, such as tumor number and diameter and the AFP level, between the two groups were also comparable. Moreover, when we compared postoperative recovery, we found most of the patients suffered no postoperative complications $(62.1 \%$ in the resection group and $61.4 \%$ in the TACE group). Due to the more rapid recovery in TACE group, the duration of hospital stay (in days) for the TACE group was significantly shorter than that for the resection group (5.1 days vs.8.2 days, $P<0.001)$. However, more patients

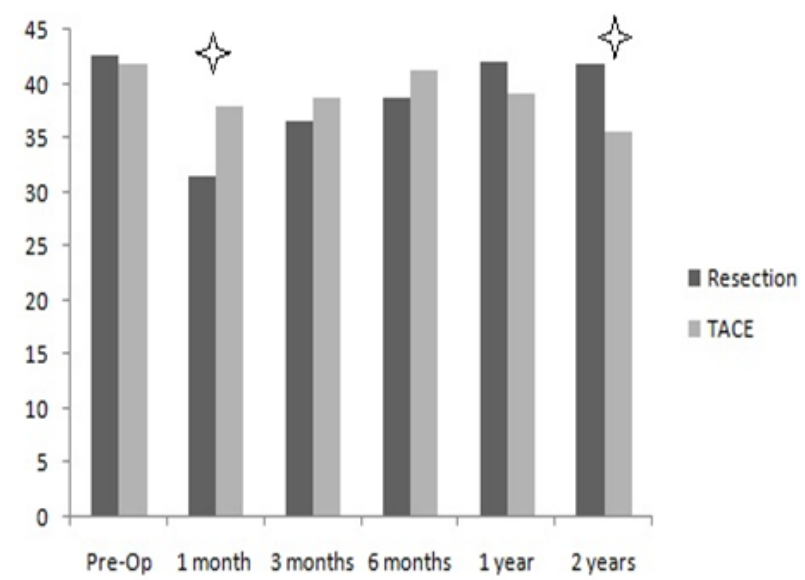

Figure 2. The MCS Score for the Resection Group was Also Significantly Lower than that for the TACE Group $(P<0.05)$, but the Resection Group Showed a Much Higher Score Compared with the TACE Group at 2 Years after Surgery (Indicated by the Stars, $P<0.05$ )

suffered HCC recurrence or metastasis in the TACE group (38.6\% in the TACE group vs.19.0\% in the resection group, $P=0.028)$.

\section{Health-related quality of life (HRQoL)}

The results of the preoperative QoL evaluation showed that the 8 domains related to QoL were all comparable between the two groups, including physical functioning $(P=0.879)$, role-physical $(P=0.632)$, bodily pain $(P=0.651)$, general health $(P=0.766)$, vitality $(P=0.452)$, social functioning $(P=0.238)$, role-emotional 
$(P=0.108)$, and mental health $(P=0.893)$. In addition, the PCS and MCS scores were comparable between the two groups at baseline (as shown in Figures 1 and 2, $P>0.05$ ).

The PCS and MCS scores were significantly decreased in both the TACE and resection groups at 1 month after surgery. However, the decreases in both the PCS and MCS scores were greater in the resection group, and these scores were significantly lower than those in the TACE group $(P<0.05)$. However, these significant differences were not present with the 3 -and 6-month post-operative questionnaires.

For the 1-year survey, the resection group showed a much higher PCS score than the TACE group $(P=0.018)$; however, the MCS scores of the two groups were not significantly different $(P=0.216)$. Further, the PCS and MCS scores on the 2-year questionnaire for the resection group were all significantly higher than those for the TACE group (as shown in Figures 1 and $2, P<0.05$ ).

\section{Tumor recurrence}

At the 2-year follow-up, 11 patients $(19.0 \%)$ in the resection group and $17(38.6 \%)$ in the TACE group suffered HCC recurrence in the liver or metastasis in nonliver organs. As shown in Table 2, the tumor recurrence rates were comparable between the resection and TACE groups at 0-1 month and 1-3 months post-operation. However, the recurrence rates were significantly higher in the TACE group than in the resection group at 3-6months, 6-12 months, and 12-24 months after surgery.

\section{Univariate and multivariateanalyses}

Additional analyses were performed to determine the risk factors forpoor QoL. The analyzed factors, which included those that are linked to QoL, were as follows: patient age, gender, BMI, cause of liver disease, Child score, initial therapy (resection or TACE), marital status, education, occupation, income, tumor number, tumor diameter, AFP level, complications, hospital stay duration in days, and recurrence. Univariate analysis identified the following factors to be predictive of poor postoperative QoL: lower education, poor income, and tumor recurrence. Multivariate Cox regression analysis was performed to assess the significant factors identified in univariate analysis and showed that tumor recurrence $(\mathrm{HR}=1.211,95 \% \mathrm{CI}: 1.086-1.415, P=0.012)$ was a significant risk factor for poor postoperative QoL among the HCC patients.

\section{Discussion}

HCC is one of the most common cancers worldwide, especially in Asian countries, due to the high prevalence of $\mathrm{HBV}$ and hepatitis $\mathrm{C}$ virus (HCV) infection. Liver resection is the most common therapy for HCC because of the shortage of liver donors, especially for early stage tumors. TACE is the most common palliative treatment for HCC. However, the choice of treatment for this disease depends not only on tumor characteristics, such as the number of tumors and their diameters, but also on liver function, which is a very important consideration in this regard (Toro et al., 2012). However, with respect to intermediate-stage $\mathrm{HCC}$ (BCLC-B), there is still controversy regarding which of these two methods provides better long-term overall survival, tumor-free survival, andtumor recurrence (Bruix et al., 2014; Zhong et al., 2014; Jianyong et al., 2015). With advances in therapies used to treat HCC, such as liver transplantation and sorafenib (Berk et al., 2013; Mancuso et al., 2015), QoL is considered to be as important as overall survival and tumor-free survival for these patients(Schipper et al., 1984); i.e., there is now a focus on not only achieving long-term survival but also patients lives' in relation to their disease and treatment (Toro Aet al., 2012). Moreover, a linear relationship was found between overall HRQoL and survival; specifically, patients reporting the highest level of overall HRQoL were found to have the longest survival time, followed by those reporting levels in the middle and lowest tertiles (Steel et al., 2014).

We found that both the PCS and MCS scores significantly decreased at 1 month after resection or TACE; however, this decrease was greater in the resection group. The TACE group may have experienced a higher QoL over the short term (less than 1 month) given that higher PCS and MCS scores were reported at the 1-month postoperative evaluation in this group compared with the liver resection group. The main reason for this finding was that trauma was caused by open-access liver resection, which led to a reduction in liver parenchyma and subsequently, to impaired liver function. Many of the resection patients' 1 month QoL scores may have been affected by pain, impaired wound healing and subjectively perceived deficiencies in their daily routines following liver resection (Bruns et al., 2010). Poon's study has revealed that the QoL after resection for HCC may increase more rapidly for patients who have undergoneminor hepatic resection compared with routine-access resection (Martin et al., 2007). These results suggest that trauma from surgery may affect short- but not long-term QoL.

Surgical treatment for HCC temporarily decreased physical QoL component, and the PCS and MCS scores began to improve at 3 months after surgery. The two groups showed comparable PCS and MCS scores, which were even lower than those determined preoperatively. A similar outcome was observed at 6 months after surgery. Moreover, our results differ from those of Mise et al. Specifically, they found a decrease in QoL following surgical treatment, which returned to baseline within 6 months, and the MCS scores reached the national average at 12 months after surgery (Mise et al., 2014). Poon et al. (2001) even demonstrated significantly greater PCS and MCS scores at 3 months after surgery for HCC compared with pre-operative scores. The main reason for these differences may be that there is a higher frequency of families lying to patients about their HCC diagnosis in China. More patients' families are likely to lie to them about their diagnosisprior to surgery. However, patients may learn the truth from other patients in the ward or other individuals. Therefore, learning their actual diagnosis may have ledto the lower post-operative PCS and MCS scores of the patients in this study compared with their preoperative scores.

With respect to long-term follow-up (6-24 months 
after surgery), the resection group had the best postoperative QoL at 24 months after surgery, and that of the TACE group peaked at 6 months after surgery. The main reason for recovery in the resection group was hepatic regeneration (which was slow in the patients with cirrhosis), an increase in liver function and only a small number of HCC recurrences. Moreover, the primary reason for the better PCS and MCS scores in the resection group over the long term may have been the low frequency of HCC recurrence. Poon et al. (2001) have noted that the removal of tumors not only improves the physical well-being of patients but also enhances their social and emotional health statuses, and their improved relationships with physicians reflecttheir satisfaction with the surgical treatment. Long-term HCC recurrence was significantly higher in the TACE group. Univariate and multivariate analyses suggested that HCC recurrence was a strong risk factor for long-term poor postoperative QoL.

There are some limitations to our present study. First, this is a single-center study that evaluated a limited number of patients. Second, the longest follow-up time was only 2 years; a longer observation period would be more convincing. Third, a psychopathological profile should be generated for a more in-depth analysis and comparison of the use of resection versus TACE for the treatment of intermediate-stage HCC patients.

In conclusion, considering the low recurrence rate and higher long-term QoL in the resection group compared with the TACE group, liver resection should be the first choice for the treatment of intermediate-stage HCC patients.

\section{References}

Jemal A, Bray F, Center MM, et al (2011). CA Cancer J Clin, 61, 69-90.

Maluccio M, Covey A (2012). Recent progress in understanding, diagnosing, and treating hepatocellular carcinoma. $C A$ Cancer J Clin, 62, 394-9.

Bruix J, Gores GJ, Mazzaferro V (2014). Hepatocellular carcinoma: clinical frontiers and perspectives. Gut, $\mathbf{6 3}$, 844-55.

Zuo CH, Xia M, Liu JS, et al (2015). Transcatheter arterial chemoembolization combined with interferon-alpha is safe and effective for patients with hepatocellular carcinoma after curative resection. Asian Pac J Cancer Prev, 16, 245-51.

Bruix J, Sherman M (2011). Management of hepatocellular carcinoma: an update. Hepatol, 53, 1020-2.

Xu C, Lv PH, Huang XE, et al (2014). Safety and efficacy of sequential transcatheter arterial chemoembolization and portal vein embolization prior to major hepatectomy for patients with HCC. Asian Pac J Cancer Prev, 15, 703-6.

Marrero JA, Fontana RJ, Barrat A, et al (2005). Prognosis of hepatocellular carcinoma: comparison of 7 staging systems in an American cohort. Hepatol, 41, 707-16.

Zhong JH, Ke Y, Gong WF, et al (2014). Hepatic resection associated with good survival for selected patients with intermediate and advanced-stage hepatocellular carcinoma. Ann Surg, 260, 329-40.

Jianyong L, Lunan Y, Wentao W, et al (2014). Barcelona clinic liver cancer stage B hepatocellular carcinoma: transarterial chemoembolization or hepatic resection? Medicine (Baltimore), 93, 180.

Slevin ML (1992). Quality of life: philosophical question or clinical reality? $B M J, \mathbf{3 0 5}, 466-9$.

Han KT, Park EC, Kim SJ, et al (2014). Factors affecting the quality of life of Korean cancer survivors who return to the workplace. Asian Pac J Cancer Prev, 15, 8783-8.

Langenhoff BS, Krabbe PF, Wobbes T, et al (2001). Quality of life as an outcome measure in surgical oncology. Br J Surg, 88, 643-52.

Scurtu R, Groza N, Otel O, et al (2005). Quality of life in patients with esophagojejunal anastomosis after total gastrectomy for cancer. Rom J Gastroenterol, 14, 367-72.

Diouf M, Bonnetain F, Barbare JC, et al (2015). Optimal cut points for quality of life questionnaire-core 30 (QLQ-C30) scales: utility for clinical trials and updates of prognostic systems in advanced hepatocellular carcinoma. Oncologist, 20, 62-71.

Diouf M, Filleron T, Barbare JC, et al (2013). The added value of quality of life (QoL) for prognosis of overall survival in patients with palliative hepatocellular carcinoma.J Hepatol, $\mathbf{5 8}, 509-21$

Yeo W, Mo FK, Koh J, et al (2006). Quality of life is predictive of survival in patients with unresectable hepatocellular carcinoma. Ann Oncol, 17, 1083-9.

Gandhi S, Khubchandani S, Iyer R (2014). Quality of life and hepatocellular carcinoma. J Gastrointest Oncol, 5, 296-317.

Fan SY, Eiser C, Ho MC (2010). Health-related quality of life in patients with hepatocellular carcinoma: a systematic review. Clin Gastroenterol Hepatol, 8, 559-64.

Bruns H, Kratschmer K, Hinz U, et al (2010). Quality of life after curative liver resection: a single center analysis. World J Gastroenterol, 16, 2388-95.

Mise Y, Satou S, Ishizawa T, et al (2014). Impact of surgery on quality of life in patients with hepatocellular carcinoma. World J Surg, 38, 958-67.

Xing M, Webber G, Prajapati HJ, et al (2015). Preservation of quality of life with doxorubicin drug eluting bead transarterial chemoembolization for unresectable hepatocellular carcinoma: longitudinal prospective study. J Gastroenterol Hepatol, [Epub ahead of print].

Wang YB, Chen MH, Yan K, et al (2007). Quality of life after radiofrequency ablation combined with transcatheter arterial chemoembolization for hepatocellular carcinoma: comparison with transcatheter arterial chemoembolization alone. Qual Life Res, 16, 389-97.

Toro A, Pulvirenti E, Palermo F, et al (2012). Health-related quality of life in patients with hepatocellular carcinoma after hepatic resection, transcatheter arterial chemoembolization, radiofrequency ablation or no treatment. Surg Oncol, 21, 23-30.

Li L, Wang H, Shen Y (2002). Development and psychometric tests of a Chinese version of the SF-36 Health Survey Scales. Zhonghua yu fang yi xue za zhi, 36, 109-13.

Jin S, Yan L, Li B, et al (2010). Quality of life and psychologic distress of recipients after adult living-donor liver transplantation (LDLT)-A study from mainland China. Transplant Proc, 42, 2611-6.

Zhang T, Zeng Y, Huang J, et al (2014). Combined resection with radiofrequency ablation for bilobar hepatocellular carcinoma: a single-center experience. J Surg Res, 191, 370-8.

Jiang L, Lei JY, Wang WT, et al (2014). Immediate radical therapy or conservative treatments when meeting the Milan criteria for advanced HCC patients after successful TACE. J Gastrointest Surg, 18, 1125-30.

Mancuso A, Mazzola A, Cabibbo G, et al (2015). Survival of patients treated with sorafenib for hepatocellular carcinoma recurrence after liver transplantation: A systematic review and meta-analysis. Dig Liver Dis, 47, 324-30

Berk V, Kaplan MA, Tonyali O, et al (2013). Efficiency and sian Pacific Journal of Cancer Prevention, Vol 16, $2015 \mathbf{4 4 5 5}$ 
ZR Xie et al

side effects of sorafenib therapy for advanced hepatocellular carcinoma: a retrospective study by the anatolian society of medical oncology. Asian Pac J Cancer Prev, 14, 7367-9.

Schipper H, Clinch J, McMurray A, et al (1984). Measuring the quality of life of cancer patients: the functional living indexcancer: development and validation.J Clin Oncol, 2, 472-83.

Steel JL, Geller DA, Robinson TL, et al (2014). Health-related quality of life as a prognostic factor in patients with advanced cancer. Cancer, 120, 3717-21.

Martin RC, Eid S, Scoggins CR, et al (2007). Health-related quality of life: return to baseline after major and minor liver resection. Surgery, 142, 676-84.

Poon RT, Fan ST, Yu WC, et al (2001). A prospective longitudinal study of quality of life after resection of hepatocellular carcinoma. Arch Surg, 136, 693-9. 